\title{
STAPHYLOCOCCUS EPIDERMIDIS NATURAL VALVE ENDOCARDITIS IN A CHILD ENDING WITH MITRAL AND AORTIC VALVES REPLACEMENT
}

\author{
Ayşe Tekin Yılmaz ${ }^{1}$, Abdülkadir Babaoğlu1ㅣ Oğuz Omay Emin Sami Arısoy ${ }^{1}$ \\ Kocaeli University Faculty of Medicine \\ 1 Department of Paediatric Health and Diseases, Kocaeli, Turkey \\ 2Department of Cardiovascular Surgery, Kocaeli, Turkey
}

\section{Introduction}

Coagulase-negative staphylococci lead to infective endocarditis mostly in patients with foreign matter such as catheter, bio-prosthesis or with immune system problems. Staphylococcus epidermidis is not an expected agent of endocarditis in previously healthy children. The present case is a scarce example of this.

Case

A 13-year old girl was admitted with fever, defatigation, tachypnea and vomiting. It was stated that she was examined three months ago for defatigation, diagnosed with iron deficiency anaemia, had been using iron medication since; the anaemia progressed nevertheless and she had a fever and vomiting for the last 4-5 days. In physical examination, her general condition was low, fever was $39^{\circ} \mathrm{C}$, peak heart rate was $175 /$ min; there was a $3 / 6$ systolic murmur and a midsystolic click at the apex. Other findings were normal. Laboratory examination revealed $7.6 \mathrm{~g} / \mathrm{dl}$ haemoglobin, $62 \mathrm{fl} \mathrm{MCV}, 24,000 / \mathrm{mm}^{3}$ leucocytes, 68.000/ $\mathrm{mm}^{3}$ thrombocytes; acute phase reactants were high. Echocardiography showed vegetation and moderate failures in aortic and mitral valves. On the third day of ceftriaxone and gentamicin treatment upon infective endocarditis diagnosis, S. epidermidis growth was found in blood cultures, vancomycin was added. The patient's fever could not be controlled; there was no regression in acute phase reactants and septic emboli developed in the finger tips and soles. On the tenth day of treatment, vegetations were surgically cleaned, the mitral and aortic valves were replaced with bio-prosthesis valves. Antibiotic treatment was continued for 6 weeks and the healthy patient was discharged to be followed-up.

\section{Conclusion}

The possibility of endocarditis must be considered in children with fever, defatigation and tachycardia. It must be kept in mind that in previously healthy children, S.epidermidis natural valve endocarditis can be devastating. 